\title{
MULTIVALUED VERSIONS OF A BOLZANO'S THEOREM
}

\author{
Jong-Sook BAe And SEOng-Hoon Cho
}

\begin{abstract}
The intermediate value theorem for a continuous real valued function is a kind of Bolzano's theorem. Similar results also hold for compact, monotone or accretive mappings in Banach spaces. In this paper we give multivalued versions of Bolzano's theorem.
\end{abstract}

\section{Introduction}

Recently Morales [17] established a close connection between a classical Bolzano's theorem from real analysis and recent works about monotone operator theory on reflexive Banach spaces. The Bolzano's theorem is a kind of intermediate value theorem stated as in Dauben [7].

Bolzano's Theorem (1817). Let $f:[-r, r] \rightarrow \mathbb{R}$ be a continuous function satisfying the following boundary condition

$$
x \cdot f(x)>0 \text { for }|x|=r .
$$

Then there exists a solution $x_{0} \in[-r, r]$ of the equation $f(x)=0$.

The existence of zeros of nonlinear mappings involving monotone operators has been widely studied. Among other things concerning the study of the existence of zeros under the boundary condition such as (1), we find the work of Vainberg and Kachurovskii [22], Minty [15, 16], Browder [5] and Shinbrot [20]. For related boundary conditions, we mention the work of Brézis et al. [3], Kachurovskii [12], Leray and Lions [14], and Rockafellar [19]. Also we remind the work of Morales [17], in which he gave a brief description of how the original problem has evolved in time passing through various generalizations for the last 30 years. However Morales [17] only studied such works for singlevalued mappings.

The goal of this paper is to give the existence of zeros of multivalued mappings, including monotone type mappings.

Received March 12, 2010.

2010 Mathematics Subject Classification. 47H10, 54H25.

Key words and phrases. fixed point, weakly inward set, monotone mapping, semimonotone mapping, strongly-monotone mapping. 


\section{Zeros of set-contractions}

Let $X$ be a Banach space. We will use $B(x, r)$ to denote the open ball centered at $x$ with radius $r$, where $x \in X$ and $r>0$. For a subset $K$ of $X, \bar{K}$ and $\partial K$ will denote the closure and the boundary of $K$, respectively. If $K$ is convex and $x \in K$, the inward set $I_{K}(x)$ of $K$ at $x$ is defined by

$$
I_{K}(x)=\{x+t(y-x) \mid y \in K, t \geq 0\} .
$$

Let $X^{*}$ be the dual space of $X$. The dual pairing $\left\langle x, x^{*}\right\rangle$ will be used for $x \in X$ and $x^{*} \in X^{*}$ instead of $x^{*}(x)$. The normalized duality mapping $J$ : $X \rightarrow 2^{X^{*}}$ is defined by

$$
J(x)=\left\{x^{*} \in X^{*} \mid\left\langle x, x^{*}\right\rangle=\|x\|^{2},\left\|x^{*}\right\|=\|x\|\right\} .
$$

Lemma 2.1. For $r>0$ and $\|x\|=r$,

$$
\bar{I}_{\bar{B}(0, r)}(x)=\cap_{x^{*} \in J(x)}\left\{w \mid\left\langle w-x, x^{*}\right\rangle \leq 0\right\} .
$$

Proof. Let $u \in I_{\bar{B}(0, r)}$ and $x^{*} \in J(x)$. Then there are $y \in \bar{B}(0, r)$ and $t \geq 0$ such that $u=x+t(y-x)$. Now compute

$$
\left\langle u-x, x^{*}\right\rangle=t\left\langle y-x, x^{*}\right\rangle \leq t\left(\|y\|\|x\|-\|x\|^{2}\right) \leq 0 .
$$

Since $\cap_{x^{*} \in J(x)}\left\{w \mid\left\langle w-x, x^{*}\right\rangle \leq 0\right\}$ is closed we conclude $\bar{I}_{\bar{B}(0, r)}(x) \subset$ $\cap_{x^{*} \in J(x)}\left\{w \mid\left\langle w-x, x^{*}\right\rangle \leq 0\right\}$.

Now assume $u \notin \bar{I}_{\bar{B}(0, r)}(x)$. Then by Hahn-Banach theorem, there exists $x^{*} \in X^{*}$ such that

$$
\left\|x^{*}\right\|=\|x\| \text { and } \sup \left\{\left\langle z, x^{*}\right\rangle: z \in \bar{I}_{\bar{B}(0, r)}(x)\right\}<\left\langle u, x^{*}\right\rangle .
$$

Then for each $\|y\| \leq r$ and $t>0$, we have

$$
\left\langle x+t(y-x), x^{*}\right\rangle<\left\langle u, x^{*}\right\rangle .
$$

This means $\left\langle y-x, x^{*}\right\rangle \leq 0$, and hence we have

$$
\left\langle x, x^{*}\right\rangle=\sup \left\{\left\langle y, x^{*}\right\rangle:\|y\| \leq r\right\}=\left\|x^{*}\right\| r=\|x\|^{2} .
$$

Finally we conclude $x^{*} \in J(x)$ and $\left\langle u-x, x^{*}\right\rangle>0$, so that $u \notin \cap_{x^{*} \in J(x)}\{w$ : $\left.\left\langle w-x, x^{*}\right\rangle \leq 0\right\}$.

To prove our results we need some fixed point theorems. First of all we mention Fan's result $[10,11]$, that every upper semicontinuous weakly inward nonempty closed convex valued multivalued mapping defined on a nonempty compact convex subset of a locally convex Hausdorff vector space has a fixed point. Let $C C(X)(K C(X))$ be the set of nonempty closed convex (compact convex, respectively) subsets of $X$.

By using Fan's result and Lemma 2.1, we have the following. 
Proposition 2.1. Let $T: \bar{B}(0, r) \rightarrow C C\left(\mathbb{R}^{n}\right)$ be an upper semicontinuous mapping such that

$$
\inf _{u \in T(x)}\langle u-x, x\rangle \leq 0 \quad \text { for } x \in \partial \bar{B}(0, r) .
$$

Then $T$ has a fixed point.

Proof. For $\epsilon>0$, let $T_{\epsilon}(x)=T(x)-\epsilon x$ for $x \in \bar{B}(0, r)$. Then for $x \in \partial \bar{B}(0, r)$,

$$
\inf _{u \in T_{\epsilon}(x)}\langle u-x, x\rangle \leq-\epsilon r^{2}<0 .
$$

Hence there exists $w \in T_{\epsilon}(x)$ such that $\langle w-x, x\rangle \leq 0$ so that $w \in \bar{I}_{\bar{B}(0, r)}(x)$ by Lemma 2.1. By Fan's result, $T_{\epsilon}$ has a fixed point, say $x_{\epsilon}$. Then we have $(1+\epsilon) x_{\epsilon} \in T\left(x_{\epsilon}\right)$. By selecting a sequence $\left\{\epsilon_{n}\right\}$ that converges to zero, we may assume that $\left\{x_{\epsilon_{n}}\right\}$ converges to some $\bar{x} \in \bar{B}(0, r)$. Then since $T$ is upper semicontinuous, we have $\bar{x} \in T(\bar{x})$.

Note that the boundary condition (2) is equivalent to the following condition:

$$
d\left(T(x), \bar{I}_{\bar{B}(0, r)}(x)\right)=0 \text { for } x \in \partial \bar{B}(0, r),
$$

where $d(A, B)=\inf \{\|a-b\| a \in A, b \in B\}$. Therefore, the method of the proof of Proposition 2.1 enables to obtain the following.

Collorary 2.1. Let $X$ be a locally convex Hausdorff topological vector space, and $K$ be a nonempty compact convex subset of $X$. Let $T: K \rightarrow C C(X)$ be an upper semicontinuous mapping satisfying

$$
d_{U}\left(T(x), \bar{I}_{K}(x)\right)=0 \text { for } x \in K
$$

for every symmetric convex neighborhood $U$ of the origin, where $d_{U}$ is the seminorm generated by $U$. Then $T$ has a fixed point.

Proof. Let $T_{U}(x)=T(x)+\bar{U}$. Then easy computation shows that $T_{U}(x) \cap$ $\bar{I}_{K}(x) \neq \emptyset$ for each $x \in K$. Hence by Fan's result, there exists a point $x_{U} \in K$ such that $x_{U} \in T\left(x_{U}\right)+\bar{U}$. Then by choosing a convergent net $\left\{x_{U}\right\}$, we can have a fixed point of $T$.

Let $\alpha$ be the Kuratowski measure of noncompactness defined on the family of bounded subsets of a Banach space $X$, and let $D$ be a bounded subset of $X$. A mapping $T: D \rightarrow K C(X)$ is a strict set-contraction if there is a constant $k<1$ such that $\alpha(T(A)) \leq k \alpha(A)$ for every $A \subset D$. Also $T$ is said to be a set-contraction if $\alpha(T(A))<\alpha(A)$ for every $A \subset D$ with $\alpha(A)>0$. According to Deimling [9] (also see [2]) every upper semicontinuous set-contractive weakly inward mapping defined on a closed convex bounded subset of a Banach space has a fixed point.

By using Lemma 2.1, we can reformulated Deimling's result as follows. 
Proposition 2.2. Let $X$ be a Banach space and $T: \bar{B}(0, r) \rightarrow X$ be an upper semicontinuous set-contraction satisfying

$$
\min _{u \in T(x)} \max _{x^{*} \in J(x)}\left\langle u-x, x^{*}\right\rangle \leq 0 \text { for } x \in \partial \bar{B}(0, r) .
$$

Then $T$ has a fixed point.

Proof. For each $x \in \partial \bar{B}(0, r)$, by (3) there exists $w \in T(x)$ such that $\langle w-$ $\left.x, x^{*}\right\rangle \leq 0$ for all $x^{*} \in J(x)$. Therefore, by Lemma 2.1, $w \in \bar{I}_{\bar{B}(0, r)}(x)$. By applying Deimling's result, we complete the proof.

In the case that the domain is not a ball the set-contractive condition of $T$ may be replaced by the strict set-contractive one and the boundary condition by that of Leray-Schauder. Here is a version of these cases, which is appeared in $[8]$.

Proposition 2.3 (Theorem 24.4, [8]). Let $D$ be a nonempty bounded open subset of a Banach space $X$, and let $T: \bar{D} \rightarrow K C(X)$ be an upper semicontinuous strict set-contraction satisfying

$$
x_{0}+\lambda\left(x-x_{0}\right) \notin T(x) \text { for } x \in \partial D, \lambda>1 \text { and some } x_{0} \in D .
$$

Then $T$ has a fixed point in $\bar{D}$.

If the domain $D$ is convex, then Proposition 2.3 is still true for set-contractive mappings.

Proposition 2.4. Let $D$ be a nonempty bounded open convex subset of a Banach space $X$, and let $T: \bar{D} \rightarrow K C(X)$ be an upper semicontinuous setcontraction satisfying condition (4). Then $T$ has a fixed point in $\bar{D}$.

Proof. Let us choose a sequence $\left\{r_{n}\right\}$ such that $0<r_{n}<1$ and $\lim r_{n}=1$. Define $T_{n}: \bar{D} \rightarrow K C(X)$ by $T_{n}(x)=\left(1-r_{n}\right) x_{0}+r_{n} T(x)$. Then each $T_{n}$ is a strict set-contraction. And also each $T_{n}$ satisfies the boundary condition (4). In fact, suppose that there are $x \in \partial D, y \in T_{n}(x)$ and $\lambda>1$ such that $y=x_{0}+\lambda\left(x-x_{0}\right)$. Then $y=\left(1-r_{n}\right) x_{0}+r_{n} u$ for some $u \in T(x)$, and hance $u=x_{0}+\lambda r_{n}^{-1}\left(x-x_{0}\right)$ where $\lambda r_{n}^{-1}>1$, which contradicts to (4).

Therefore by Proposition 2.3, we have $x_{n} \in T_{n}\left(x_{n}\right)$ for some $x_{n} \in \bar{D}$. Now choose $y_{n} \in T\left(x_{n}\right)$ such that $x_{n}=\left(1-r_{n}\right) x_{0}+r_{n} y_{n}$. Then an easy computation gives $\alpha\left(\left\{y_{n}\right\}\right)=\alpha\left(\left\{x_{n}\right\}\right)$. But since $F$ is a set-contraction, we have $\alpha\left(\left\{x_{n}\right\}\right)=$ 0 .

Otherwise we have

$$
\alpha\left(\left\{x_{n}\right\}\right)=\alpha\left(\left\{y_{n}\right\}\right) \leq \alpha\left(T\left(\left\{x_{n}\right\}\right)\right)<\alpha\left(\left\{x_{n}\right\}\right),
$$

which is a contradiction.

Now we may assume $\left\{x_{n}\right\}$ converges to some $\bar{x} \in \bar{D}$, so that $\left\{y_{n}\right\}$ also converges to $\bar{x}$. Since $T$ is upper semicontinuous we have $\bar{x} \in T(\bar{x})$. 
Proposition 2.5. Let $F: \bar{B}(0, r) \rightarrow C C\left(\mathbb{R}^{n}\right)$ be an upper semicontinuous mapping satisfying

$$
\sup _{y \in F(x)}\langle y, x\rangle \geq \delta \text { for all } x \in \partial \bar{B}(0, r),
$$

where $\delta>0$. Then $\bar{B}\left(0, \frac{\delta}{r}\right) \subset F(\bar{B}(0, r))$. In particular, the equation $0 \in F(x)$ has a solution in $\bar{B}(0, r)$.

Proof. Let $v \in \bar{B}\left(0, \frac{\delta}{r}\right)$ and define $T(x)=x+v-F(x)$ for $x \in \bar{B}(0, r)$. Now compute for $x \in \partial \bar{B}(0, r)$

$$
\begin{aligned}
\inf _{u \in T(x)}\langle u-x, x\rangle & =\inf _{y \in F(x)}\langle v-y, x\rangle \\
& =\langle v, x\rangle-\sup _{y \in F(x)}\langle y, x\rangle \\
& \leq \frac{\delta}{r} \cdot r-\delta=0 .
\end{aligned}
$$

By Proposition 2.1, we have a fixed point $\bar{x}$ in $\bar{B}(0, r)$ of $T$. Since $\bar{x} \in$ $\bar{x}+v-F(\bar{x})$, we have $v \in F(\bar{x})$.

Our first multivalued version of Bolzano's theorem is the following theorem.

Theorem 2.2. Let $F: \bar{B}(0, r) \rightarrow C C\left(\mathbb{R}^{n}\right)$ be an upper semicontinuous mapping satisfying

$$
\sup _{y \in F(x)}\langle y, x\rangle \geq 0 \text { for all } x \in \partial \bar{B}(0, r) .
$$

Then the equation $0 \in F(x)$ has a solution in $\bar{B}(0, r)$.

Proof. For $\epsilon>0$, let $F_{\epsilon}(x)=\epsilon x+F(x)$. Then by Proposition 2.5, we have an $x_{\epsilon} \in \bar{B}(0, r)$ such that $0 \in \epsilon x_{\epsilon}+F\left(x_{\epsilon}\right)$. By choosing a sequence $\left\{\epsilon_{n}\right\}$ converging to 0 and $\left\{x_{\epsilon_{n}}\right\}$ converges to some $\bar{x} \in \bar{B}(0, r)$, we can obtain $0 \in F(\bar{x})$.

We note that the condition (5) is equivalent to the condition

$$
d\left(x-F(x), \bar{I}_{\bar{B}(0, r)}(x)\right)=0 \text { for all } x \in \partial \bar{B}(0, r) .
$$

By applying Corollary 2.1, directly, $T(x)=x-F(x)$ has a fixed point. Also note that if $F$ is singlevalued, then Theorem 2.2 reduces to Theorem 1 of [17].

In general Banach spaces, the domain of a given mapping may not be expected to be compact. In this case, we need some compactness conditions on the mapping to obtain our results.

Here is an analogous result of Proposition 2.5.

Proposition 2.6. Let $X$ be a Banach space and let $F: \bar{B}(0, r) \rightarrow K C(X)$ be an upper semicontinuous mapping satisfying $T(x)=x-F(x)$ is a setcontraction, and

$$
\max _{y \in F(x)} \min _{x^{*} \in J(x)}\left\langle y, x^{*}\right\rangle \geq \delta \text { for all } x \in \partial \bar{B}(0, r) \text { and some } \delta>0 .
$$


Then $\bar{B}\left(0, \frac{\delta}{r}\right) \subset F(\bar{B}(0, r))$. In particular, the equation $0 \in F(x)$ has a solution in $\bar{B}(0, r)$.

Proof. For $v \in \bar{B}\left(0, \frac{\delta}{r}\right)$, define $T_{v}(x)=x+v-F(x)$. Then as in the proof of Proposition 2.5, one can easily show that $\min _{u \in T_{v}(x)} \max _{x^{*} \in J(x)}\left\langle u-x, x^{*}\right\rangle \leq 0$ for each $x \in \partial \bar{B}(0, r)$. Therefore by applying Proposition 2.2, we have an $\bar{x} \in \bar{B}(0, r)$ such that $\bar{x} \in \bar{x}+v-F(x)$, so that $v \in F(\bar{x})$.

As a consequence of Proposition 2.6 obtain a multivalued version of Corollary 2 in [17] in general Banach spaces, which is an extension to Hilbert spaces of what Lax [13] calls the intermediate value theorem.

Collorary 2.3. Let $X$ be a Banach space and let $F: \bar{B}(0, r) \rightarrow K C(X)$ be an upper semicontinuous mapping satisfying $T(x)=x-F(x)$ is a set-contraction, and

$$
x \in F(x) \text { for } x \in \partial \bar{B}(0, r) .
$$

Then $\bar{B}(0, r) \subset F(\bar{B}(0, r))$.

Proof. For $x \in \partial \bar{B}(0, r)$,

$$
\max _{y \in F(x)} \min _{x^{*} \in J(x)}\left\langle y, x^{*}\right\rangle \geq \min _{x^{*} \in J(x)}\left\langle x, x^{*}\right\rangle \geq r^{2} .
$$

Therefore, by Proposition 2.6, we have $\bar{B}(0, r) \subset F(\bar{B}(0, r))$.

Next we give a Banach space version of Theorem 2.2 as follows.

Theorem 2.4. Let $X$ be a Banach space and let $F: \bar{B}(0, r) \rightarrow K C(X)$ be an upper semicontinuous mapping satisfying $T(x)=x-F(x)$ is a set-contraction, and

$$
\max _{y \in F(x)} \min _{x^{*} \in J(x)}\left\langle y, x^{*}\right\rangle \geq 0 \text { for all } x \in \partial \bar{B}(0, r) .
$$

Then the equation $0 \in T(x)$ has a solution in $\bar{B}(0, r)$.

Proof. One can easily show that the condition (6) implies the boundary condition (3). Hence by Proposition 2.2, T has a fixed point $\bar{x}$ in $\bar{B}(0, r)$, so that $0 \in F(\bar{x})$.

Next we give the second multivalued version of the Bolzano's theorem. We begin with bounded convex domains whose interior is nonempty.

Theorem 2.5. Let $D$ be a nonempty bounded open convex subset of a Banach space $X$ with $0 \in D$. Suppose $F: \bar{D} \rightarrow K C(X)$ is an upper semicontinuous mapping satisfying $T(x)=x-F(x)$ is a set-contraction, and

$$
\min _{y \in F(x)} \max _{x^{*} \in J(x)}\left\langle y, x^{*}\right\rangle \geq 0 \text { for all } x \in \partial D .
$$

Then the equation $0 \in F(x)$ has a solution in $\bar{D}$. 
Proof. It suffices to show that $T$ satisfies the condition (4) for $x_{0}=0$. In fact, assume that $\lambda x \in T(x)$ for some $x \in \partial D$ and $\lambda>1$. Then $\lambda x=x-y$ for some $y \in F(x)$. For each $x^{*} \in J(x)$, we have

$$
\left\langle y, x^{*}\right\rangle=(1-\lambda)\|x\|^{2}<0,
$$

which contradicts to (7).

Then by applying Proposition 2.4, $\bar{x} \in T(\bar{x})$ for some $\bar{x} \in D$, that is, $0 \in$ $F(\bar{x})$.

In Theorem 2.5, if $T(x)=x-F(x)$ is a strict set-contraction, then the convexity condition can be omitted.

Theorem 2.6. Let $D$ be a nonempty bounded open subset of a Banach space $X$ with $0 \in D$. Suppose that $F: \bar{D} \rightarrow K C(X)$ is an upper semicontinuous mapping satisfying:

(i) $T(x)=x-F(x)$ is a strict set-contraction;

(ii) the boundary condition (7) holds.

Then the equation $0 \in F(x)$ has a solution in $\bar{D}$.

Proof. As in the proof Theorem 2.5, (ii) implies the boundary condition (4). By applying Proposition 2.3, we have a fixed point of $T$, which is a solution of the equation $0 \in T(x)$.

\section{Zeros of monotone mappings}

A natural question is whether one can replace the strict set-contractive condition by a different type of condition. As a result monotonicity conditions have captured a great deal of interest to solve the equations (see $[3,12,15,16,19]$ ).

Let $D$ be a subset of a (real) Banach space $X$, and let $F: D \rightarrow 2^{X^{*}}$ be a multivalued mapping. Then

$F$ is said to be monotone if, for all $x, y \in D$

$$
\langle y-x, v-u\rangle \geq 0 \text { for all } u \in F(x), v \in F(y) .
$$

$F$ is said to be semi-monotone if, for all $x, y \in D$ and $u \in F(x)$, there is $v \in F(y)$ such that

$$
\langle y-x, v-u\rangle \geq 0 .
$$

For example, if $T$ is a multivalued nonexpansive mapping defined on a subset of a Hilbert space, then $I-T$ is semi-monotone (see [1]).

$F$ is said to be strongly-monotone if there exists a constant $c>0$ such that for all $x, y \in D, u \in F(x)$ and $v \in F(y)$

$$
\langle y-x, v-u\rangle \geq c\|y-x\|^{2} .
$$

The following lemma comes from definitions directly. 
Lemma 3.1. Let $F: D \subset X \rightarrow K C\left(X^{*}\right)$.

(i) $F$ is monotone if and only if for all $x, y \in D$,

$$
\max _{u \in F(x)}\langle y-x, u\rangle \leq \min _{v \in F(y)}\langle y-x, v\rangle .
$$

(ii) $F$ is semi-monotone if and only if for all $x, y \in D$,

$$
\min _{u \in F(x)}\langle y-x, u\rangle \leq \min _{v \in F(y)}\langle y-x, v\rangle .
$$

The following lemma comes from Lemma 1 in [1].

Lemma 3.2. Let $X$ be a (real) Banach space, $D$ a nonempty bounded subset of $X$ and let $C$ be a nonempty (strongly) compact subset of $X^{*}$. Then the function

$$
f(x)=\min _{u \in C}\langle x, u\rangle
$$

is weakly uniformly continuous on $D$.

The following variational inequality is well known and comes from KKM theory.

Proposition 3.1. Let $K$ be a nonempty compact convex subset of a Hausdorff topological vector space. Let $\phi$ and $\psi$ be two real-valued functions defined on $K \times K$ satisfying

(a) $\phi(x, y) \leq \psi(x, y)$ for all $(x, y) \in K \times K$, and $\psi(x, x) \leq 0$ for all $x \in K$; $K$

(b) for each fixed $x \in K, \phi(x, y)$ is a lower semicontinuous function of $y$ on

(c) for each fixed $y \in K$, the set $\{x \in X \mid \psi(x, y)>0\}$ is convex.

Then there exists a point $y_{0} \in K$ such that $\phi\left(x, y_{0}\right) \leq 0$ for all $x \in K$.

Proof. Apply Theorem 2 of [1] for $X=X_{0}=K$.

Throughout the last part of this paper we may assume that the reflexive Banach space $X$ and its dual $X^{*}$ are both locally uniformly convex after renorming (see [21]). This fact implies that the normalized duality mapping $J$ is singlevalued and strictly monotone.

Theorem 3.1. Let $X$ be a reflexive (real) Banach space, and let $G$ be a bounded open subset of $X$ with $0 \in G$. Suppose that $F: \overline{c o}(G) \rightarrow K C\left(X^{*}\right)$ is semimonotone and upper semicontinuous along line segments on $\overline{c o}(G)$ to the weak* topology on $X^{*}$ satisfying

$$
\min _{u \in F(x)}\langle x, u\rangle>0 \text { for all } x \in \partial G .
$$

Then the equation $0 \in F(x)$ has a solution in $G$.

Proof. Let $K=\overline{c o}(G)$ and let us define $\phi, \psi: K \times K \rightarrow R$ by

$$
\phi(x, y)=\min _{u \in F(x)}\langle y-x, u\rangle
$$




$$
\psi(x, y)=\min _{v \in F(y)}\langle y-x, v\rangle .
$$

Now equip $X$ with the weak topology. Then all conditions (a)-(c) in Proposition 3.1 are satisfied. Actually, (a) comes from semi-monotonicity of $F$ and Lemma 3.1(ii), and (b) comes from Lemma 3.2. Also an easy computation gives the condition (c).

Therefore by Proposition 3.1, there exists a point $y_{0} \in K=\overline{c o}(G)$, such that

$$
\phi\left(x, y_{0}\right)=\min _{u \in F(x)}\left\langle y_{0}-x, u\right\rangle \leq 0 \text { for all } x \in K .
$$

Next we claim that

$$
\min _{v \in F\left(y_{0}\right)}\left\langle y_{0}-x, v\right\rangle \leq 0 \text { for all } x \in K .
$$

To prove (10), let $x \in K$ be fixed. Define $z_{t}=t x+(1-t) y_{0}$ for $t \in[0,1]$. Since $K$ is convex, each $z_{t} \in K$. By applying (9), we get

$$
\min _{u \in F\left(z_{t}\right)}\left\langle y_{0}-z_{t}, u\right\rangle=t \min _{u \in F\left(z_{t}\right)}\left\langle y_{0}-x, u\right\rangle \leq 0 \text { for all } t \in(0,1],
$$

and hence we get

$$
\min _{u \in F\left(z_{t}\right)}\left\langle y_{0}-x, u\right\rangle \leq 0 \text { for all } t \in(0,1]
$$

On the contrary, assume $\min _{v \in F\left(y_{0}\right)}\left\langle y_{0}-x, v\right\rangle>0$. Then $F\left(y_{0}\right)$ is a subset of weak* open set $U=\left\{w \in X^{*} \mid\left\langle y_{0}-x, w\right\rangle>0\right\}$. Since $F$ is upper semicontinuous along line segments to the weak ${ }^{*}$ topology, there is some $t_{0} \in(0,1)$ such that $F\left(z_{t_{0}}\right) \subset U$. As $F\left(z_{t_{0}}\right)$ is compact,

$$
\min _{u \in F\left(z_{t_{0}}\right)}\left\langle y_{0}-x, u\right\rangle>0
$$

which contradicts to (11). Therefore (10) is true.

Now we will prove $y_{0} \in G$. If $y_{0} \notin G$, then $y_{0}=\lambda y$ for some $y \in \partial G$ and $\lambda \geq 1$. If $\lambda=1$, then because of $0 \in G$, by (10) we have

$$
\min _{v \in F\left(y_{0}\right)}\left\langle y_{0}, v\right\rangle \leq 0
$$

which contradicts to (8). If $\lambda>1$, then by (9) we have

$$
\min _{u \in F(y)}\left\langle y_{0}-y, u\right\rangle=(\lambda-1) \min _{u \in F(y)}\langle y, u\rangle \leq 0,
$$

which also contradicts to (8). Therefore we conclude $y_{0} \in G$.

Since $G$ is open, from (10) we can prove

$$
\min _{v \in F\left(y_{0}\right)}\langle x, v\rangle \leq 0 \text { for all } x \in X .
$$

Indeed, if $x \in X$, then $y_{0}-t x \in G$ for some $t>0$. By (10), we have

$$
\min _{v \in F\left(y_{0}\right)}\left\langle y_{0}-\left(y_{0}-t x\right), v\right\rangle \leq 0 .
$$

Since $t>0$, we have $\min _{v \in F\left(y_{0}\right)}\langle x, v\rangle \leq 0$. 
Since $X$ is reflexive, by Hahn-Banach theorem, we know that (12) implies $0 \in F\left(y_{0}\right)$.

Since every monotone mapping is semimonotone, we have the following corollary.

Collorary 3.2. Let $X$ be a reflexive (real) Banach space, and let $G$ be a bounded open subset of $X$ with $0 \in G$. Suppose that $F: \overline{c o}(G) \rightarrow K C\left(X^{*}\right)$ is monotone and upper semicontinuous along line segments on $\overline{c o}(G)$ to the weak* topology on $X^{*}$ satisfying condition (8). Then the equation $0 \in F(x)$ has a solution in $G$.

Theorem 3.3. Let $X$ be a reflexive (real) Banach space, and let $G$ be a bounded open subset of $X$ with $0 \in G$. Suppose that $F: \overline{c o}(G) \rightarrow K C\left(X^{*}\right)$ is monotone and upper semicontinuous satisfying

$$
\min _{u \in F(x)}\langle x, u\rangle \geq 0 \text { for all } x \in \partial G .
$$

Then the equation $0 \in F(x)$ has a solution in $\bar{G}$.

Proof. Let $F_{\epsilon}=F+\epsilon J$, where $J$ is the duality mapping and $\epsilon>0$. Then $F_{\epsilon}$ satisfies the boundary condition (8) on $\partial G$, and $F_{\epsilon}$ is still monotone. Therefore by Corollary 3.2, the equation $0 \in F_{\epsilon}(x)$ has a solution in $G$.

By selecting a sequence $\left\{\epsilon_{n}\right\}$ in $(0,1)$ that converges to zero, we find a sequence $\left\{x_{n}\right\}$ in $G$ such that

$$
0 \in F\left(x_{n}\right)+\epsilon_{n} J\left(x_{n}\right) .
$$

Therefore there exists a sequence $\left\{y_{n}\right\}$ such that $y_{n} \in F\left(x_{n}\right)$ and $y_{n}+$ $\epsilon_{n} J\left(x_{n}\right)=0$ for all $n \geq 1$. Since $F$ is monotone, for all $m, n$

$$
\left\langle x_{m}-x_{n}, y_{m}-y_{n}\right\rangle \geq 0 \text {, }
$$

and so we get

$$
\left\langle x_{m}-x_{n}, \epsilon_{m} J\left(x_{m}\right)-\epsilon_{n} J\left(x_{n}\right)\right\rangle \leq 0 .
$$

Then as in the proof of Lemma 1 of [17], we conclude $x_{n} \rightarrow x$ for some $x \in \bar{G}$. In this case since $y_{n} \rightarrow 0$, we have $0 \in F(x)$ by the upper semicontinuity of $F$.

The boundary condition (8) can be weakened in Corollary 3.2 if $F$ is lower semicontinuous.

Proposition 3.2. Let $X$ be a reflexive (real) Banach space, and let $G$ be a bounded open subset of $X$ with $0 \in G$. Suppose that $F: \overline{c o}(G) \rightarrow K C\left(X^{*}\right)$ is monotone and lower semicontinuous along line segments on $\overline{c o}(G)$ to the weak ${ }^{*}$ topology on $X^{*}$ satisfying

$$
\max _{u \in F(x)}\langle x, u\rangle>0 \text { for all } x \in \partial G .
$$

Then the equation $0 \in F(x)$ has a solution in $G$. 
Proof. Essentially the process of the proof are similar as in the proof of Theorem 3.1 .

Let $K=\overline{c o}(G)$ and for $(x, y) \in K \times K$,

$$
\phi(x, y)=\max _{u \in F(x)}\langle y-x, u\rangle, \psi(x, y)=\min _{v \in F(y)}\langle y-x, v\rangle .
$$

Then by Lemma 3.1(i), we can apply Proposition 3.1 such that there exists a point $y_{0} \in K$ satisfying

$$
\phi\left(x, y_{0}\right)=\max _{u \in F(x)}\left\langle y_{0}-x, u\right\rangle \leq 0 \text { for all } x \in K .
$$

Now we claim that

$$
\max _{v \in F\left(y_{0}\right)}\left\langle y_{0}-x, v\right\rangle \leq 0 \text { for all } x \in K .
$$

To prove (15), let $x \in K$ be fixed. Define $z_{t}=t x+(1-t) y_{0}$ for $t \in[0,1]$. Since each $z_{t} \in K$. By applying (14), we get

$$
\max _{u \in F\left(z_{t}\right)}\left\langle y_{0}-z_{t}, u\right\rangle=t \max _{u \in F\left(z_{t}\right)}\left\langle y_{0}-x, u\right\rangle \leq 0 \text { for all } t \in(0,1],
$$

and hence we have

$$
\max _{u \in F\left(z_{t}\right)}\left\langle y_{0}-x, u\right\rangle \leq 0 \text { for all } t \in(0,1] .
$$

Suppose that (15) dose not hold. Then we have $\max _{v \in F\left(y_{0}\right)}\left\langle y_{0}-x, v\right\rangle>0$, so that $F\left(y_{0}\right) \cap U \neq \emptyset$, where $U=\left\{w \in X^{*} \mid\left\langle y_{0}-x, w\right\rangle>0\right\}$ is a weak ${ }^{*}$ open set of $X^{*}$. Since $F$ is lower semicontinuous along line segments on $\overline{c o}(G)$ to the weak* topology on $X^{*}$, there is some $t_{0} \in(0,1)$ such that $F\left(z_{t_{0}}\right) \cap U \neq \emptyset$. As a result we have

$$
\max _{u \in F\left(z_{t_{0}}\right)}\left\langle y_{0}-x, u\right\rangle>0
$$

which contradicts to (16). Therefore (15) holds. As in the proof of Theorem 3.1 , by applying (13) and (15), one can easily prove $y_{0} \in G$. Above all, since $\min _{u \in F(x)}\left\langle y_{0}-x, u\right\rangle \leq \max _{u \in F(x)}\left\langle y_{0}-x, u\right\rangle$, inequalities (9) and (10) still hold. Therefore by the same way as in the proof of Proposition 3.1, we can prove $0 \in F\left(y_{0}\right)$.

Analogous to Theorem 3.3, we obtain the following theorem and the proof is essentially same.

Theorem 3.4. Let $X$ be a reflexive (real) Banach space, and let $G$ be a bounded open subset of $X$ with $0 \in G$. Suppose that $F: \overline{c o}(G) \rightarrow K C\left(X^{*}\right)$ is monotone and continuous from $\overline{c o}(G)$ to weak* topology on $X^{*}$ satisfying

$$
\max _{u \in F(x)}\langle x, u\rangle \geq 0 \text { for all } x \in \partial G \text {. }
$$

Then the equation $0 \in F(x)$ has a solution in $\bar{G}$. 
Domain invariance theorems for singlevalued strongly-monotone mappings were studied by many authors (see $[4,15,17,18]$ ). By using Corollary 3.2 or Proposition 3.2, we will obtain an invariance of domain theorem for locally strongly-monotone multivalued mappings.

Theorem 3.5. Let $G$ be an open subset of a reflexive (real) Banach space $X$, and let $F: G \rightarrow K C\left(X^{*}\right)$ be a locally strongly-monotone mapping. If $F$ is either upper semicontinuous or lower semicontinuous along line segments on $G$ to the weak ${ }^{*}$ topology on $X^{*}$, then $F(G)$ is open in $X^{*}$.

Proof. Let $z_{0} \in G$ and $w_{0} \in F\left(z_{0}\right)$. By letting $\bar{F}(z)=F\left(z+z_{0}\right)-w_{0}$, we may assume that $0 \in G$ and $0 \in F(0)$. Since $F$ is locally strongly-monotone, there exists $r>0$ such that $\bar{B}(0, r) \subset G$ and for some $c>0$

$$
\langle y-x, v-u\rangle \geq c\|y-x\|^{2} \text { for all } x, y \in \bar{B}(0, r), u \in F(x), v \in F(y) .
$$

Let $w \in X^{*}$ with $\|w\|<c r$, and let $F_{1}(z)=F(z)-w$ for $z \in \bar{B}(0, r)$. For $x \in \partial \bar{B}(0, r)$, we have

$$
\begin{aligned}
\max _{u \in F_{1}(x)}\langle x, u\rangle & \geq \min _{u \in F_{1}(x)}\langle x, u\rangle \\
& =\min _{u \in F(x)}\langle x, u-w\rangle \\
& =\min _{u \in F(x)}\{\langle x-0, u-0\rangle-\langle x, w\rangle\} \\
& \geq c\|x\|^{2}-\|x\|\|w\|>0 .
\end{aligned}
$$

By applying Corollary 3.2 or Proposition 3.2, we know that $w \in F(z)$ has a solution in $B(0, r)$. Hence $B(0, c r) \subset F(B(0, r))$, which shows that $F(G)$ is open.

\section{References}

[1] J. S. Bae, W. K. Kim, and K. K. Tan, Another generalization of Ky Fan's minimax inequality and its applications, Bull. Inst. Math. Acad. Sinica 21 (1993), no. 3, 229-244.

[2] J. S. Bae and M. S. Park, Fixed points of $\mu$-condensing maps with inwardness conditions, Math. Japon. 40 (1994), no. 1, 179-183.

[3] H. Brézis, M. Crandall, and A. Pazy, Perturbations of nonlinear maximal monotone sets in Banach space, Comm. Pure Appl. Math. 23 (1970), 123-144.

[4] F. E. Browder, Variational boundary value problems for quasi-linear elliptic equations of arbitrary order, Proc. Nat. Acad. Sci. U.S.A. 50 (1963), 31-37.

[5] Existence of periodic solutions for nonlinear equations of evolution, Proc. Nat. Acad. Sci. U.S.A. 53 (1965), 1100-1103.

[6] _ Remarks on nonlinear functional equations III, Illinois J. Math. 9 (1965), 617622 .

[7] J. W. Dauben, Progress of mathematics in the early 19th century: context, contents and consequences, Impact of Bolzano's epoch on the development of science (Prague, 1981), 223-260, Acta Hist. Rerum Nat. necnon Tech. Spec. Issue, 13, ČSAV, Prague, 1982.

[8] K. Deimling, Nonlinear Functional Analysis, Springer-Verlag, Berlin, 1985.

[9] _ Fixed points of weakly inward multis, Nonlinear Anal. 10 (1986), no. 11, 12611262. 
[10] Ky Fan, A generalization of Tychonoff's fixed point theorem, Math. Ann. 142 (1961), 305-310.

[11] _ Some properties of convex sets related to fixed point theorems, Math. Ann. 266 (1984), no. 4, 519-537.

[12] R. I. Kachurovskii, Monotonic nonlinear operators in Banach spaces, Dokl. Akad. Nauk SSSR 163 (1965), 559-562.

[13] P. D. Lax, Change of variables in multiple integrals, Amer. Math. Monthly 106 (1999), no. 6, 497-501.

[14] J. Leray and J. L. Lions, Quelques résulatats de Višik sur les problèmes elliptiques nonlinéaires par les méthodes de Minty-Browder, Bull. Soc. Math. France 93 (1965), 97-107.

[15] G. J. Minty, Monotone (nonlinear) operators in Hilbert space, Duke Math. J. 29 (1962), $341-346$.

[16] G. J. Minty, On a "monotonicity" method for the solution of non-linear equations in Banach spaces, Proc. Nat. Acad. Sci. U.S.A. 50 (1963), 1038-1041.

[17] C. H. Morales, A Bolzano's theorem in the new millennium, Nonlinear Anal. 51 (2002), no. 4, Ser. A: Theory Methods, 679-691.

[18] J. A. Park, Invariance of domain theorem for demicontinuous mappings of type $\left(S_{+}\right)$, Bull. Korean Math. Soc. 29 (1992), no. 1, 81-87.

[19] R. Rockafellar, Local boundedness of nonlinear, monotone operators, Michigan Math. J. 16 (1969), 397-407.

[20] M. Shinbrot, A fixed point theorem, and some applications, Arch. Rational Mech. Anal. 17 (1964), 255-271.

[21] S. L. Trojanski, On locally uniformly convex and differentiable norms in certain nonseparable Banach spaces, Studia Math. 37 (1971), 173-180.

[22] M. M. Vainberg and R. I. Kachurovskii, On the variational theory of non-linear operators and equations, Dokl. Akad. Nauk SSSR 129 (1959), 1199-1202.

JONG-SOOK BAE

Department of Mathematics

MOYNGJI UNIVERSITY

YONGIN 449-728, KOREA

E-mail address: jsbae@mju.ac.kr

SEOng-Hoon Cho

Department of Mathematics

HANSEO UNIVERSITY

SEOSAN 356-706, KorEA

E-mail address: shcho@hanseo.ac.kr 